
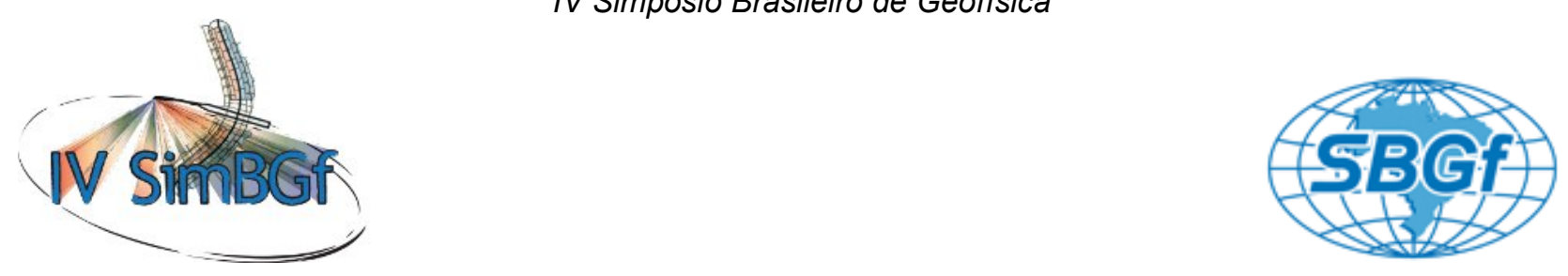

\title{
Resultados de prospecção geofísica aplicada à mineração de calcário.
} Jorel Yuri Dechiche, Vagner Roberto Elis, Giorgio de Tomi, Álvaro Rezende, USP

Copyright 2010, SBGf - Sociedade Brasileira de Geofísica

Este texto foi preparado para a apresentação no IV Simpósio Brasileiro de Geofísica, Brasilia, 14 a 17 de novembro de 2010. Seu conteúdo foi revisado pelo Comitê Técnico do IV SimBGf, mas não necessariamente representa a opinião da SBGf ou de seus associados. É proibida a reprodução total ou parcial deste material para propósitos comerciais sem prévia autorização da SBGf

\section{Resumo}

Este trabalho expõe resultados do emprego do método geoelétrico para sondagens em mineração de calcário, como teste dessa metodologia no contexto do planejamento de lavra.

\section{Introdução}

Uma empresa mineradora, cujo nome é omitido por um critério de confidencialidade, solicitou ao LAPOL (sigla para Laboratório de Planejamento e Organização de Lavra) do Departamento de Engenharia de Minas e de Petróleo da Escola Politécnica da USP um modelo de reservatório mineral que foi construído pela interpolação de dados produzidos por poços de sondagem direta. Diante disso o LAPOL decidiu realizar testes de sondagem indireta (i.e. geofísicas) para adensar a malha do modelo preexistente formulado com dados obtidos da perfuração. Conseqüentemente foram sugeridas as técnicas sondagem elétrica vertical associada com medidas polarização induzida.

Esse trabalho tem o objetivo de expor resultados de uma aquisição de dados elétricos que foram produzidos pelo método de polarização induzida associado ao método de sondagem elétrica vertical.

O objetivo dessas sondagens foi avaliar a possibilidade de emprego de tal metodologia geofísica para a caracterização e detecção de interfaces de camadas geológicas no contexto da mineração de calcário.

\section{Sondagens geoelétricas teste de resultados.}

Houve a necessidade de fazer testes de sondagem elétrica para verificar se as técnicas geofísicas são aplicáveis ao contexto geológico e as necessidades de exploração da mina. Conforme ocorre a abertura de novas frentes de lavra em uma mineração surge à necessidade de conhecimento do volume do reservatório mineral e da espessura da capa de solo. A técnica geofísica empregada nesse teste foi sondagem elétrica vertical de resistividade e polarização induzida.

A sondagem elétrica vertical ou SEV consiste em uma sucessão de medidas de um parâmetro físico, a resistividade aparente (método da eletrorresistividade), efetuadas com o mesmo tipo de arranjo e separação crescente entre os eletrodos de emissão e recepção, mantendo-se fixos o centro do arranjo (ponto de atribuição do ensaio) e sua orientação.

Ao introduzir uma corrente elétrica (I) através dos eletrodos $\mathrm{A}$ e $\mathrm{B}$, e entre os eletrodos $\mathrm{M}$ e $\mathrm{N}$ medir a diferença de potencial criada $(\Delta \mathrm{V})$, resulta que as medidas pertinentes serão utilizadas para o cálculo da resistividade aparente pela fórmula:

$$
\rho_{\mathrm{a}}=\mathrm{K} \cdot \frac{\Delta \mathrm{V}}{\mathrm{I}} \text { (ohm.m) }
$$

Sendo $\mathrm{K}$ um fator geométrico que só depende do espaçamento entre os quatro eletrodos.

$$
\mathrm{K}=\pi \cdot \frac{(\overline{\mathrm{AM}} \cdot \overline{\mathrm{AN}})}{\overline{\mathrm{MN}}}
$$

Aumentando-se a distância entre os eletrodos de corrente $\mathrm{A}$ e $\mathrm{B}$, o volume total da subsuperfície incluída na medida também aumenta, permitindo alcançar camadas cada vez mais profundas. Os resultados sucessivos estarão, portanto, estritamente ligados com as variações da resistividade com a profundidade.

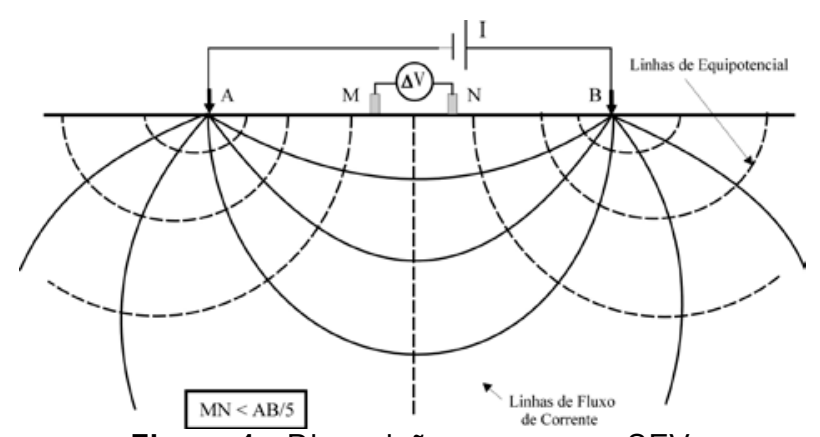

Figura 1 - Disposição no campo - SEV

A polarização induzida, ao se aplicar, por intermédio de eletrodos de corrente denominados convencionalmente de $A$ e $B$ cravados na superfície do terreno, uma 
diferença de potencial $\Delta \mathrm{V}$ primária ao solo, é provocada conseqüentemente uma polarização do mesmo.

Essa diferença de potencial primária $(\Delta V p)$ não se estabelece e nem se anula instantaneamente quando a corrente é emitida e cortada em pulsos sucessivos. Ela varia com o tempo na forma de uma curva $\Delta V_{\mathbb{I P}}=f(t)$. Esta curva, liga a assíntota $\Delta \mathrm{Vp}$ em regime estacionário com a assíntota zero após o corte da corrente (Figura 1). $\mathrm{A}$ amplitude de um valor $\Delta \mathrm{V}_{\mathrm{IP}}(\mathrm{t})$ está diretamente ligada à maior ou menor capacidade que os terrenos têm de se polarizarem, constituindo-se, portanto, na base do método. Esta capacidade de polarização constitui a susceptibilidade IP dos materiais da terra.

Analogamente, pode-se descrever o fenômeno da polarização induzida como se solo contivesse pequenos condensadores que se carregariam durante a emissão de corrente, descarregando-se após o corte. $\mathrm{A}$ curva $\Delta \mathrm{V}_{\mathrm{IP}}=$ $f(t)$ poderia ser chamada então de curva de descarga IP.

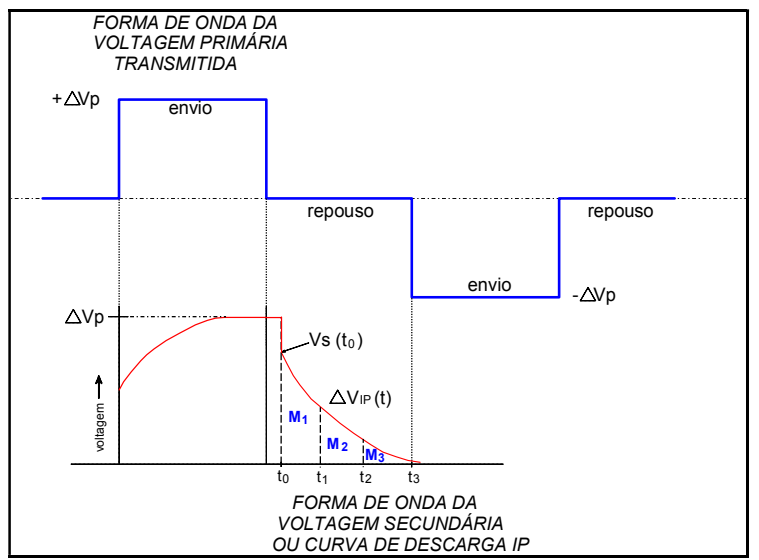

Figura 2 - Curva de descarga IP-Domínio do Tempo.

$$
\mathrm{M}=\frac{\Delta \mathrm{V}_{\mathrm{IP}} \times 1000}{\Delta \mathrm{V}_{\mathrm{P}}} \quad(\mathrm{mV} / \mathrm{V})
$$

Como ocorre no método da eletrorresistividade, se as medidas da polarização induzida são efetuadas sobre um terreno cujo subsolo é homogêneo, quaisquer das magnitudes definidas acima podem ser utilizadas como medida de sua polarização verdadeira. Na prática, como o meio pode ser considerado heterogêneo, o resultado das medidas dependerá da posição dos eletrodos que compõem o arranjo de campo utilizado em relação às heterogeneidades polarizáveis.

Resulta, portanto, que os dados de campo expressam polarizações aparentes, que no presente trabalho, como será utilizado o IP-Domínio do Tempo, o parâmetro resultante das medidas será denominado de cargabilidade aparente (Ma), que será o parâmetro utilizado para a interpretação, que é realizada em conjunto com a resistividade.

Foram feitas sondagens elétricas com ambas as técnicas SEV e IP em quatro furos de sondagem em diferentes pontos da área de exploração mecânica com testemunhos catalogados. Devido ao fato de o calcário ser mais suscetível a alteração que o gnaisse, espera-se resistividade um pouco menores para este tipo em relação ao gnaisse. Porém o contraste de resistividade pode não ser suficiente para sua diferenciação nos ensaios de resistividade. Por isso, foram realizados ensaios de polarização induzida em conjunto, para auxiliar na identificação do contato.

\section{Resultados}

As SEVs 1 e 2 foram executadas em uma parte da mina onde ocorre calcário sobre gnaisse. $O$ objetivo foi verificar a eficiência da geofísica na identificação da transição solo - calcário e da base do calcário em contato com o gnaisse.

A primeira SEV foi executada na Linha 450 , onde os dados do poço (F458/02) identificaram 9,1 metros de solo e o contato calcário - gnaisse em 71,2 metros. O resultado dessa SEV é apresentado na Figura 3, onde pode-se que o solo de alteração ocorre até 9,5 metros, caracterizado por resistividade inferior a 33 ohm.m e baixa cargabilidade. O calcário inalterado ocorre de 9,5 a 59,0 metros, com resistividade de 535 ohm.m e alta cargabilidade (33 mV/V). Abaixo desse nível, ocorre o gnaisse com resistividade maior que o calcário (10.500 ohm.m) e cargabilidade de $4,4 \mathrm{mV} / \mathrm{V}$.

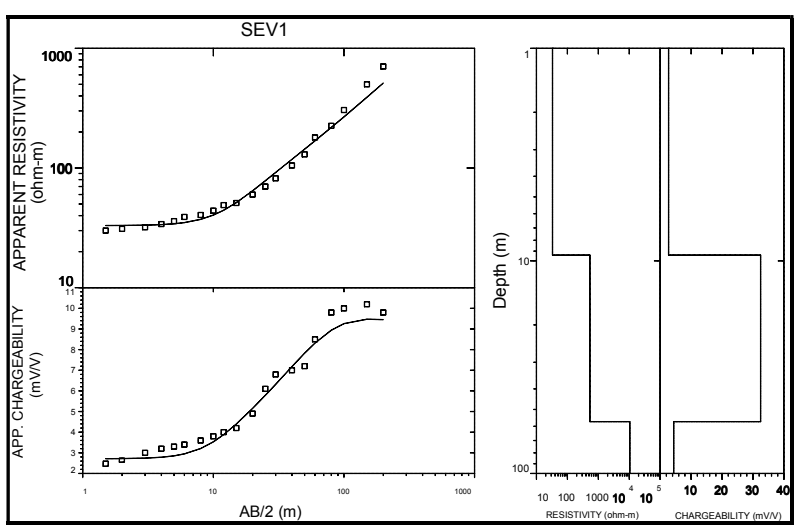

\begin{tabular}{|c|c|c|c|c|}
\hline Camada & $\begin{array}{c}\text { Espessura } \\
(\mathbf{m})\end{array}$ & $\mathbf{\rho}\left(\mathrm{ohm}_{\mathbf{w}} \mathbf{m}\right)$ & $\mathbf{M}(\mathbf{m V} / \mathbf{V})$ & Interpretação \\
\hline 1 & 9,5 & 33 & 2,7 & Solo de alteracăãa \\
\hline 2 & 49,5 & 535 & 33,0 & Calcário \\
\hline 3 & & 10500 & 4,4 & Gnaisse \\
\hline
\end{tabular}

Figura 3 - SEV realizada na Linha 450.

A segunda SEV foi executada na Linha 800 , onde os dados do poço (F792/03) identificaram 27,1 metros de solo e o contato calcário - gnaisse em 83.9 metros. O resultado desta SEV é apresentado na Figura 4, onde pode-se observar um modelo de cinco camadas. As três primeiras caracterizam, respectivamente, o solo superficial (47 ohm.m, 1,6 mV/V), seguida de solo não saturado (270 ohm.m, 6,1 mV/V) e o solo de alteração abaixo da zona saturada (29 ohm.m, $50 \mathrm{mV} / \mathrm{V}$ ). O 
calcário inalterado apresenta maior resistividade (1300 ohm.m) e cargabilidade de $61 \mathrm{mV} / \mathrm{V}$, a partir de 26,3 metros de profundidade. O gnaisse ocorre a partir de 87,3 metros de profundidade, com 10.980 ohm.m e 2 $\mathrm{mV} / \mathrm{V}$.

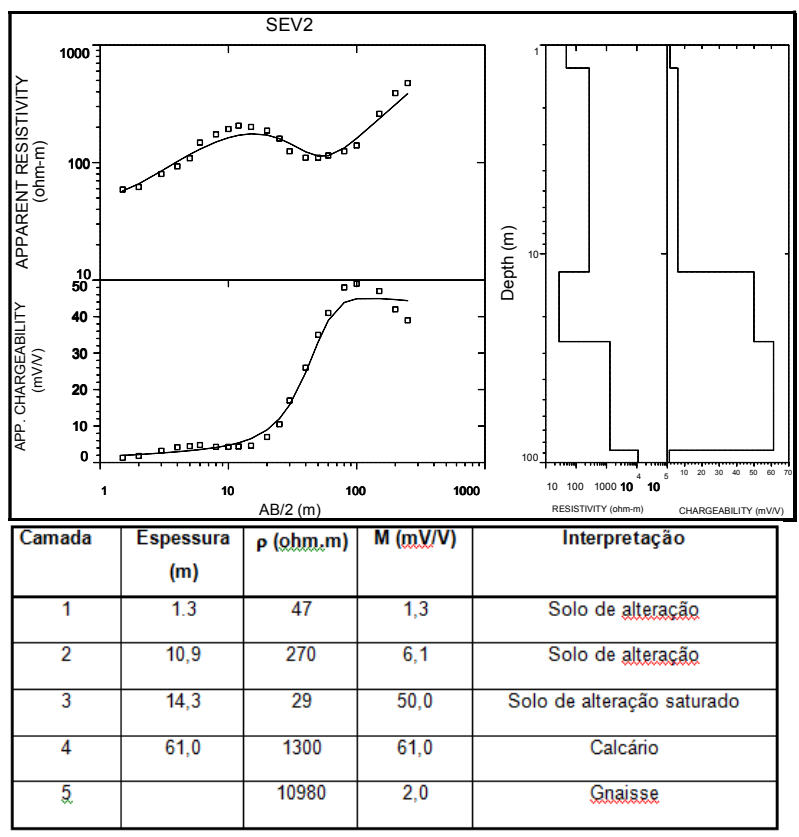

Figura 4 - SEV realizada na Linha 800.

As SEVs 3 e 4 foram executadas em outra parte da mina onde ocorre uma camada de gnaisse sobre o calcário, que assenta-se sobre o gnaisse inferior. $O$ objetivo foi verificar a eficiência da geofísica na identificação da transição solo - gnaisse superior e dos contatos do calcário com os gnaisses superior e inferior.

A SEV3 foi executada próxima do poço F01-02, onde os dados do poço (F458/02), cuja descrição é apresentada na Figura 5:

\begin{tabular}{|l|c|}
\hline Profundidade (m) & Descrição litológica \\
\hline $0-3,45$ & Solo (gnaisse) \\
\hline $3,45-46,0$ & Gnaisse \\
\hline $46,0-62,0$ & Calcário \\
\hline
\end{tabular}

Figura 5 - Descrição do poço F01-02

O resultado da terceira SEV é apresentado na Figura 6, onde pode-se observar que não houve uma correlação satisfatória com o perfil do poço. Nessa SEV, os estratos superiores com resistividades entre 122 e 172 ohm.m e baixas cargabilidades $(0,1$ a $1,2 \mathrm{mV} / \mathrm{V})$ são interpretados como solo de alteração de gnaisse e gnaisse alterado. Essas camadas ocorrem na SEV até 23,6 metros, ao passo que no furo ocorrem até 46,0 metros. Essa diferença pode ser explicada pelo mergulho do contato que influenciará os dados geofísicos, o que evidentemente não acontece com o dado do poço. $A$ seguir ocorre uma camada com 970 ohm.m e 9,2 mV/V, valores compatíveis com o calcário da Área Magna. Por isso, esta camada é interpretada como calcário e ocorre de 23,6 a 86,5 metros. A partir de 86,5 metros ocorre uma camada muito resistividade e de baixa cargabilidade (14.895 ohm.m e 0,4 mV/V, compatível com o gnaisse inferior observado nas SEVs 1 e 2.

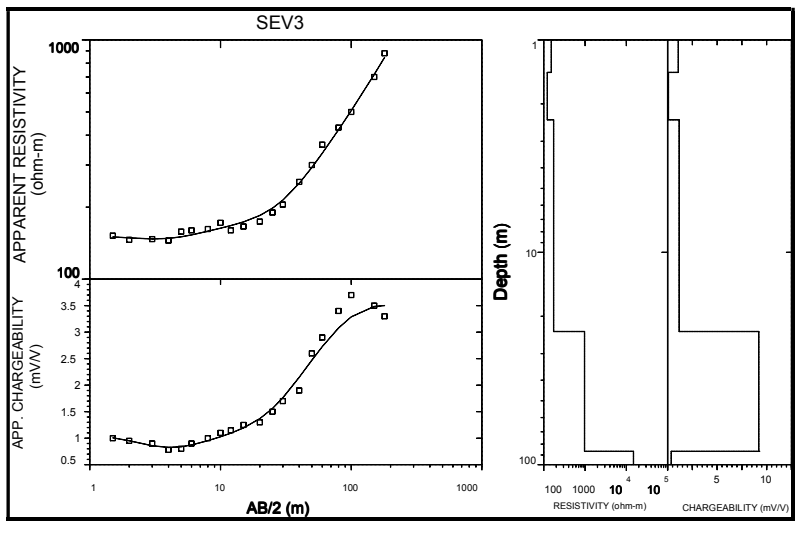

\begin{tabular}{|c|c|c|c|c|}
\hline Camada & $\begin{array}{c}\text { Profundidade } \\
(\mathbf{m})\end{array}$ & $\boldsymbol{\rho}(\mathrm{ohm}, \mathrm{m})$ & $\mathrm{M}(\mathrm{mV} / \mathrm{V})$ & Interpretaçäo \\
\hline 1 & $0-1,4$ & 152 & 1,1 & Solo de alteração (gnaisse) \\
\hline 2 & $1,4-2,4$ & 122 & 0,1 & Solo de alteração (gnaisse) \\
\hline 3 & $2,4-23,6$ & 172 & 1,2 & Gnaisse alterado \\
\hline 4 & $23,6-86,5$ & 970 & 9,2 & Calcáro \\
\hline 5 & $86,5-$ & 14895 & 0,4 & Gnaisse inferior \\
\hline
\end{tabular}

Figura 6 - SEV3 realizada próxima ao poço F01-02.

A quarta SEV foi executada na posição do poço F98-21. Os dados deste poço são apresentados na Figura 7.

\begin{tabular}{|l|c|}
\hline Profundidade $(\mathbf{m})$ & Descrição litológica \\
\hline $0-22,9$ & Solo (gnaisse) \\
\hline $22,9-55,6$ & $\begin{array}{c}\text { Gnaisse migmatisse migmatito com } \\
\text { intercalações quartzosas }\end{array}$ \\
\hline $55,6-62,0$ & Calcário \\
\hline $62,0-103,7$ & \\
\hline
\end{tabular}

Figura 7 - Descrição do poço F98-21

O resultado da SEV4 é apresentado na Figura 8, onde pode-se observar que também não houve uma correlação satisfatória com o perfil do poço. Nessa SEV, os estratos superiores com resistividades entre 88 e 204 ohm.m e baixas cargabilidades (1,8 a 2,6 mV/V) são interpretados como solo de alteração de gnaisse e 
gnaisse alterado. Essas camadas ocorrem na SEV até 10,3 metros, ao passo que no furo ocorrem até 62,0 metros. Nesse caso, a incerteza na interpretação aumentou e a explicação da diferença devido ao mergulho do contato é muito arriscada. A seguir ocorre uma camada com 486 ohm.m e $11,5 \mathrm{mV} / \mathrm{V}$, seguida de outra com 545 ohm.m e 3,8 mV/V. Os valores de resistividade são compatíveis com o calcário da Área 1 , onde foram executadas as SEVs 1 e 2, e embora os de cargabilidade sejam menores que os esperados, ainda são superiores aos do gnaisse inferior. Por isso, estas camadas são interpretadas como calcário, que ocorre de 10,3 a 88,6 metros. A partir de 88,6 metros ocorre uma camada muito resistiva e de baixa cargabilidade (16.720 ohm.m e $0,02 \mathrm{mV} / \mathrm{V}$, compatível com o gnaisse inferior observado nas SEVs 1 e 2.

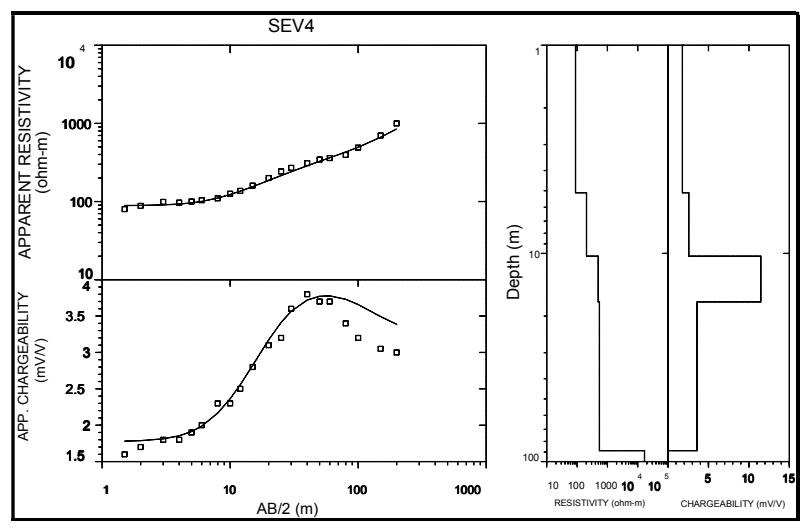

\begin{tabular}{|c|c|c|c|c|}
\hline Camada & $\begin{array}{c}\text { Profundidade } \\
(\mathbf{m})\end{array}$ & $\mathbf{\rho}$ (ohm,m) & $\mathrm{M}(\mathrm{mV} / \mathrm{V})$ & Interpretaçăo \\
\hline 1 & $0-5,1$ & 88 & 1,8 & Solo de alteração (gnaisse) \\
\hline 2 & $5,1-10,3$ & 204 & 2,6 & Gnaisse alterado \\
\hline 3 & $10,3-17,7$ & 496 & 11,5 & Calcário \\
\hline 4 & $17,7-88,6$ & 545 & 3,8 & Calcário \\
\hline 5 & $88,6-$ & 16720 & 0,02 & Gnaisse inferior \\
\hline
\end{tabular}

Figura 8 - SEV4 realizada próxima ao poço F98-21.

\section{Discussão e Conclusões}

Estes resultados mostram que a identificação dos contrastes de interesse sofrem muitas limitações, em especial na identificação do contato do calcário com o gnaisse superior. Por outro lado, pode-se dizer que foi possível identificar o contato inferior entre calcário e gnaisse, baseados nos estudos realizados na Área 1. As diferenças entre as interfaces calcário-gnaisse nas SEVs em relação aos poços podem ser explicada pela limitação da técnica geofísica na identificação de contatos com topografia altamente irregular e mergulho acentuado. Como a técnica foi desenvolvida para estratos planoparalelos e topografia plana, quanto mais as condições de campo se afastaram disso, maior tenderá a ser a diferença entre os dados reais e o modelo geoelétrico. Os resultados obtidos nos testes preliminares mostram que a metodologia pode ser utilizada com sucesso na identificação do contato inferior, como forma de adensar a malha de amostragem na mina. Ainda sugere-se que alguns ensaios sejam realizados diretamente sobre área de ocorrência de gnaisse (sem calcário recobrindo) para garantir maior segurança na interpretação dos dados.

\section{Agradecimentos}

Laboratório de Planejamento e Organização de Lavra (LAPOL-USP), Sociedade Extrativa Dolomia LTDA, Casadei Engenharia e Departamento de Geofísica (IAGUSP).

\section{Referências}

Burguer, H. R., 1992. Exploration geophysics of the shallow subsurface. Prentice Hall (Ed.). Englewood Ciffs), New Jersey. Pg: 241-316.

Keller, G.V \& Frischknecht, 1966. Electrical methods in geophysical prospecting, Pergamon Press, Head Hill Hall, Oxford, UK pg: 90-196.

Pursell, E.M., 1964. Berkeley physics course- volume 2. Mcgraw-hill book company, New York, USA, vol. 2: 110294. 